incoordination, and sensori-perception impairments ("minimal brain dysfunction" or MBD) is an important part of the work-up of a child at risk of ADD. The omission of signs of impaired motor control and perception from the diagnostic criteria for ADHD excludes many ADD children with significant classroom dysfunction. A return to the former, more objective MBD criteria, in addition to the subjective, symptomatic criteria for ADHD, would lead to the earlier recognition of children with increased risk of classroom dysfunction sufficient to demand medical, psychological, and educational therapy. See Progress in Pediatric Neurology III, PNB Publ, 1997;pp204-205, for a review of previous Scandinavian reports of the DAMP syndrome and ADHD.

Huttenlocher PR (1990) and Millichap JG (1974) have emphasized the value of neurologic soft signs in the prediction of learning disabilities in preschool children and the correlation with hyperactive behavior and response to methylphenidate. (see Progress in Pediatric Neurology I, 1991;pp167-8).

\title{
ATTENTION DEFICITS, ADHD, AND COMPLEX PARTIAL SEIZURES
}

The degree of attention deficits in children with complex partial seizures (CPS), with and without ADHD, were compared with that found in children with ADHD but without epilepsy, in a study at the Department of Educational Psychology, University of Texas, Austin. A computerized performance test (CPT), evaluating sustained attention, inhibition of response, response time, and consistency of response, was completed by 12 children with CPS and ADHD, 21 with CPS alone, 22 with ADHD alone, and 15 controls. CPT performance was unrelated to IQ scores on the WISC-R. Children with CPS and ADHD had the lowest scores on the CPT. Children with CPS had impaired sustained attention regardless of the diagnosis of ADHD. Antiepileptic medication, usually carbamazepine, taken by $78 \%$ of the patients with CPS was considered an unlikely cause of the attention deficits. Methylphenidate administered to patients with ADHD improved performance of the CPT in both the group with seizures and without. (Semrud-Clikeman M, Wical B. Components of attention in children with complex partial seizures with and without ADHD. Epilepsia February 1999;40:211-215). (Reprints: Dr M Semrud-Clikeman, Department of Educational Psychology, SZB 504, University of Texas, Austin, TX 78413).

COMMENT. Complex partial epilepsy is associated with attention problems that interfere with learning and memory. In patients with CPE complicated by ADHD, methylphenidate has a similar beneficial effect on attention as that observed in children with ADHD without seizures.

An EEG is indicated in children with ADHD who have episodes of confusion or staring that interfere with attention and learning. Treatment with carbamazepine may be advisable before the initiation and addition of stimulant medication. CBZ-induced lowering of MPH blood levels is reported (see p.15).

\section{ARACHNOID CYST DECOMPRESSION AND COGNITIVE RECOVERY}

A 20-year-old right-handed male with a large left temporal arachnoid cyst, discovered incidentally by CT following a kick to the head with brief loss of consciousness, is reported from the University of Texas Medical Branch, Galvestan, TX. MRI revealed a mass effect with midline shift and left temporal lobe hypoplasia. Severe headaches had increased in frequency for one year after the head trauma. Early history and developmental milestones were normal, but learning disabilities and behavior disorder lead to school drop-out at 11 th grade. Pre-surgery psychological testing revealed a borderline verbal IQ of 76 and average performance IQ of 90, with clinical discrepancy in verbal (left hemisphere) and visual-perceptual/constructional (right hemisphere) skills. 
Bilateral deficits were observed on a test of speed of motor coordination. School records were comparable to the post-trauma test scores. Craniotomy with cyst fenestration and cystoperitoneal shunt resulted in minimal change in size of cyst. Psychological testing at 1 year postsurgery showed no change in language function but significant cognitive improvements in verbal learning, memory, visual-perception/constructional skills, and psychomotor speed, with a 27-point increase in Performance IQ to 117. Reliance on anatomical decompression of the arachnoid cyst may underestimate the efficacy of surgical intervention. (Soukup VM, Patterson J, Trier TT, Chen JW. Cognitive improvement despite minimal arachnoid cyst decompression. Brain Dev Dec 1998;20:589-593). (Respond: Dr Vicki M Soukup, Department of Neurology, University of Texas Medical Branch, Galveston, TX 77555).

COMMENT. Surgical intervention in certain cases of left temporal arachnoid cyst can result in improved cognitive function despite only marginal reduction in size of the cyst. In addition to refractory seizures and headaches, cognitive measures should be used as a functional index of surgical intervention and outcome.

Neurosurgeons in general are reluctant to operate on arachnoid cysts, except in cases complicated by mass effect with seizures and headaches. The report of a Temporal Lobe Arachnoid Cyst/ADHD syndrome, described recently in children with coincidental learning and language disabilities, has emphasized the importance of tests for associated attention deficits and learning disorders in children with arachnoid cysts located in the temporal fossa. (Millichap JG. Temporal lobe arachnoid cyst-attention deficit disorder syndrome. Neurology May 1997;48:1435-1439). PET studies have sometimes demonstrated hypometabolism in cortical areas adjacent to a cyst, and cystoperitoneal shunt can result in improved metabolism and language function. A neurobiologic basis for ADHD, suggested by the TLAC/ADHD syndrome, may prove amenable to surgery in cases of arachnoid cyst complicated by symptoms of a mass effect.

\section{MPH-INDUCED CHANGES IN RIGHT FRONTAL METABOLISM}

The effects of methylphenidate (MPH), a drug that increases dopamine, on brain glucose metabolism in 20 cocaine abusers was studied using PET scans and measurements of D2 receptors at the Brookhaven National Laboratory, the State University of New York at Stony Brook, and New York University, NY. MPH increased metabolism in the superior cingulate, right thalamus, and cerebellum. MPH-induced changes in the right striatum and orbitofrontal cortex were associated with cocaine craving, and those in the prefrontal cortex were associated with mood. Compulsive drug abuse in addicted persons may be related to activation of the right striatum and orbitofrontal cortex, areas known to be abnormal in compulsive disorders. The predominant correlation of craving with right but not left brain regions warrants further investigation of laterality of drug responses. (Volkow ND, Wang G-J, Fowler JS et al. Association of methylphenidate-induced craving with changes in right striato-orbitofrontal metabolism in cocaine abusers: Implications in addiction. Am I Psychiatry Jan 1999;156:19-26). (Reprints: Dr Nora D Volkow, Medical Department, Brookhaven National Laboratory, Bldg 490, Upton, NY 11973).

COMMENT. In normal subjects, these authors have reported variable cerebral metabolic responses to MPH; some showing increased metabolism, some decreases, and some no change. Metabolic responses were correlated with measures of dopamine D2 receptors. Similarly, in cocaine abusers, metabolic responses were correlated with dopamine receptors; those with higher D2 levels 